\title{
Experimental study of columns partially filled with concrete under compressive axial loads
}

\section{Etude expérimentale des colonnes partiellement remplis par le béton sous charge axiale}

\author{
N. Handel ${ }^{1}$, N.-E. Chiekh ${ }^{2}$, and D. Achoura ${ }^{3}$ \\ ${ }^{1}$ LGMRIU, Département de génie civil, Université Mohamed Cherif Messaadia Souk-Ahras, Algérie \\ ${ }^{2}$ LMDC, Département de génie civil, Université Mentouri Constantine, Algérie \\ ${ }^{3}$ LGC, Département de génie civil, Université Badji Mokhtar Annaba, Algérie
}

\begin{abstract}
In the present work, results of tests conducted on thin welded steel-concrete stubs are presented. A total of 24 stubs an I steel section were tested under axial compression at 28 days after the date of casting, 4 were empty, 4 filled with normal concrete, 8 columns had shear connecters welded along the centreline of the web, and 8 columns had steel rods welded between the tips of opposing flanges on both sides of the spacing of the transverse link $100 \mathrm{~mm}$ and $50 \mathrm{~mm}$. The main parameters studied were: the heel height, and type of connector strengthening. From the test results, it is confirmed that the thin walls are more sensitive to the appearance local buckling and the length of the profiles has a significant effect on the bearing capacity and failure mode. The bearing capacity was increased with the addition of strengthening connectors.
\end{abstract}

Résumé. Dans cette étude, on présente les résultats expérimentaux obtenus sur des poteaux mixtes béton-acier mince réalisés par soudures. Un total de 24 profilés en acier, et en forme de I a été testé sous charge de compression uni-axiale à l'âge de 28 jours. les spécimens ont été réparties comme suit: 4 à vides, 4 partiellement remplies avec un béton ordinaire sans l'addition des connecteurs, 4 renforcés par des connecteurs de cisaillements de type cornière en U, 4 autres l'ont été avec des connecteurs de cisaillements type goujons et 8 restants ont été renforcés avec des liens transversaux d'espacement $100 \mathrm{~mm}, 50 \mathrm{~mm}$, soudés aux bouts des ailes opposées. Les principaux paramètres étudiés sont: l'élancement du profilé, le type de connecteur de renforcement. A partir des résultats d'essais obtenus, il est confirmé que les parois minces sont plus sensibles de l'apparition au voilement et la longueur des profilés a un effet considérable sur la capacité portante et le mode de rupture. L'addition des connecteurs de renforcement a confirmé l'augmentation de la charge ultime par rapport aux profilés sans connecteurs. 


\section{Introduction}

Il est connu que les poteaux mixtes partiellement ou totalement enrobés de béton, ou les profilés creux remplis de béton, combinent les avantages du béton et de l'acier de construction, à savoir : la vitesse de construction, la capacité portante élevée, la résistance au feu et le poids de l'acier léger. Actuellement, il y a un grand nombre d'études sur les colonnes tubulaires en acier remplies de béton ; cependant, peu de recherche a été conduite sur des profilés remplis partiellement de béton. Tremblay et al. [1] Chicoine.T et al. [2] ; Bouchereau et Toupin [3]; Begum et al[4], Brent. S. P et Robert G. D [5], Handel. N et al.[6], ont étudié le comportement et la capacité portante d'un nouveau type de colonne composée partiellement remplie avec un béton ordinaire faite avec section en I à parois minces et soudée, forcée avec des liens transversaux. Le mode de rupture des spécimens était le voilement local avec déformation de l'acier au niveau des ailes et l'écrasement du béton, les études ont montré que l'utilisation des renforts additionnels peut améliorer le comportement du profilé et peut augmenter la charge ultime du poteau.

Une série d'essais a été effectuée sur 24 profilés, partagés en deux groupes. Dans le premier groupe, 8 profilés ont été répartis comme suit : 4 à vides, 4 partiellement remplis avec un béton ordinaire sans l'addition des connecteurs. Dans le second groupe, un total de 16 profilés partiellement enrobés, ont été testés. Les spécimens testés comportent : 4 renforcés par des connecteurs de cisaillements de type cornière en U soudées le long de la ligne centrale de l'âme, 4 autres l'ont été avec des connecteurs de cisaillements type goujons, soudées le long de la ligne centrale de l'âme, et les 8 restants ont été renforcés avec des liens transversaux d'espacement $100 \mathrm{~mm}, 50 \mathrm{~mm}$, soudés aux bouts des ailes opposées pour pouvoir augmenter le confinement du béton.

Les paramètres étudiés sont l'influence de la hauteur du profilé (200 à $500 \mathrm{~mm}$ ) et l'effet du type de connecteur de renforcement. Les résultats expérimentaux enregistrés ont été comparés avec ceux donnés par la prescription du règlement EC4 pour les profilés composés.

\section{Programme experimental}

\subsection{Descriptions des spécimens}

Un total de 24 poteaux, de section transversale $(100 \times 70 \times 2) \mathrm{mm}$ a été étudié. L'acier utilisé pour la confection de la section transversale en I est sous forme d'une tôle mince galvanisée et laminée à froid d'épaisseur de $2 \mathrm{~mm}$ et obtenue par façonnage et pliage à froid pour former des U. La section étudiée est fabriquée par 2 aciers laminés à froid initialement en forme de $\mathrm{U}$ et soudés dos à dos avec une soudure continue sur toute la hauteur du spécimen pour former une section d'acier reconstituée en forme de I "Figure 1". Le rapport de largeur de l'aile sur l'épaisseur (b/t) pour tous les spécimens est de 17.50; les élancements des éprouvettes sont : 200-300-400-500 mm. Le premier connecteur de cisaillement est de type cornière en forme de $U$ de section $25 \times 25 \mathrm{~mm}$ et d'épaisseur $8 \mathrm{~mm}$, Le deuxième connecteur est de type goujon de diamètre $8 \mathrm{~mm}$ et de longueur $32 \mathrm{~mm}$. Ces deux connecteurs de cisaillement sont soudés au centre de l'âme horizontalement, et sont verticalement espacés de $100 \mathrm{~mm}$ le long de la hauteur du profilé. La troisième solution utilisée pour le renforcement des profilés en acier partiellement enrobés, consiste à des liens horizontaux en acier rond de forme $\mathrm{U}$ de diamètre $3,3 \mathrm{~mm}$, soudé sur les bouts des semelles. L'espacement des liens horizontaux est de $100 \mathrm{~mm}$ et $50 \mathrm{~mm}$. 


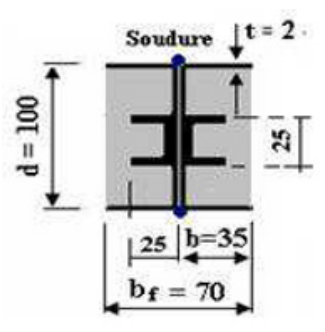

(a)

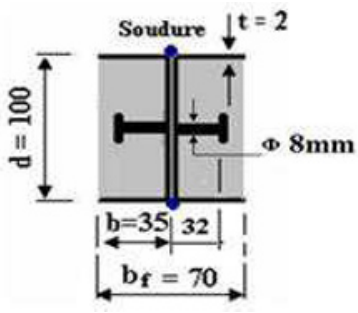

(b)

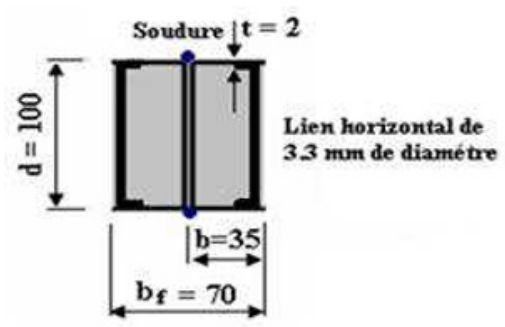

(c)

Fig. 1. Sections reconstituées transversales en I renforcées par: (a) cornière en U, (b) goujon, (c) lien horizontal.

\subsection{Caractéristique de l'acier utilisé}

Les caractéristiques mécaniques de l'acier utilisé pour la confection des profilés sont :

- Module de Young: $\mathrm{Ea}=205000 \mathrm{MPaDo}$ not add any page numbers to your paper.

- Contrainte d'écoulement: $\sigma_{\mathrm{e}}=300 \mathrm{MPa}$

\subsection{Composition du béton utilisé}

La méthode utilisée pour déterminer la composition du béton de remplissage est celle de GorisseDreux. Elle a été calculée pour un diamètre maximum des granulats égal à $10 \mathrm{~mm}$ et un affaissement de $6 \mathrm{~cm}$ correspondant à un béton plastique. La composition du béton est comme suit :

- Dosage du ciment CPJ 42,5 = 350Kg/m3 ;

- Rapport eau sur ciment $=0.6$, sable $0 / 2,5=811,32 \mathrm{Kg} / \mathrm{m} 3$;

- Gravier de calcaire $5 / 10=1095,77 \mathrm{Kg} / \mathrm{m} 3$;

- Masse volumique $=2,47 \mathrm{Kg} / \mathrm{m} 3$;

- Résistance moyenne des cubes de béton à l'âge de 28 jours est fc28 = 26 MPa.

\subsection{Coulage}

Les 24 poteaux en acier remplis partiellement de béton ordinaire, ont été coulés en position verticale et par 3 couches, chacune compactée sur une table vibrante pendant 2 à 3 minutes pour réaliser une meilleure uniformité du béton le long de la longueur du spécimen. Les poteaux composés sont remplis à partir de la même gâché; 6 cubes de 100x100x100 mm ont été également coulés pendant le remplissage des poteaux. Après 24 heures on a décoffré les spécimens préparés. Ils sont posés à l'environnement de laboratoire à une température ambiante $\left(\mathrm{T}=20-25^{\circ} \mathrm{C}\right.$ et $\left.\mathrm{HR}=60 \mathrm{à} 70 \%\right)$ pendant 28 jours.

\subsection{Matériel d'essai et procédure}

Tous les spécimens ont été testés sur une machine de compression de capacité $500 \mathrm{KN}$, sous compression uni-axiale à une vitesse de chargement d'ordre de $0.6 \mathrm{Tf} / \mathrm{s}$. Une attention particulière a été observée pour vérifier la position correcte des spécimens avant n'importe quel chargement. Les faces supérieures et inférieures des poteaux composés sont traités mécaniquement pour éliminer les irrégularités de surfaçage, et pour s'assurer que la charge appliquée est répartie simultanément à travers la section transversale sur ses composants acier et béton. 


\section{Résultats des essais}

Un total de 24 profilés en acier remplis par le béton ordinaire, sont testés sous compression axiale. La charge de rupture est atteinte lorsqu'il n'y a plus possibilité de toute augmentation du chargement et que le mécanisme de rupture commence à prendre place. Toutes les données et les dimensions des spécimens testés ont été récapitulés dans le tableau1.

Tableau 1. Dimensions des poteaux testés.

\begin{tabular}{|c|c|c|c|c|c|}
\hline Test séries & Spécimen & $\begin{array}{c}\mathrm{bf} \\
(\mathrm{mm})\end{array}$ & $\begin{array}{c}\mathrm{d} \\
(\mathrm{mm})\end{array}$ & $\begin{array}{c}\text { Epaiss } \\
\text { Acier t } \\
(\mathrm{mm})\end{array}$ & $\begin{array}{c}\text { hauteur du } \\
\text { specimen L } \\
(\mathrm{mm})\end{array}$ \\
\hline Série 0 & PV 200 & 69,00 & 101,60 & 1,91 & 196,00 \\
(profilé vide) & PV 300 & 69,50 & 99,55 & 1,99 & 298,38 \\
& PV 400 & 68,63 & 100,50 & 2,00 & 397,50 \\
& PV 500 & 68,75 & 100,63 & 1,99 & 495,25 \\
\hline & $\mathrm{P} 1 / 200$ & 69,20 & 104,30 & 2,03 & 195,50 \\
Série 1 & $\mathrm{P} 1 / 300$ & 68,55 & 100,50 & 2,04 & 298,25 \\
(sans connecteur) & $\mathrm{P} 1 / 400$ & 69,50 & 99,62 & 1,99 & 396,37 \\
& $\mathrm{P} 1 / 500$ & 68,50 & 101,37 & 2,01 & 449,50 \\
\hline & $\mathrm{P} 2 / 200$ & 68.60 & 100.03 & 1.93 & 198.20 \\
Série 2 & $\mathrm{P} 2 / 300$ & 69.03 & 100.18 & 1.98 & 287.50 \\
(avec connecteur cornière) $)$ & $\mathrm{P} 2 / 400$ & 68.83 & 101.08 & 1.93 & 378.75 \\
& $\mathrm{P} 2 / 500$ & 68.43 & 101.50 & 2.00 & 477.50 \\
\hline & $\mathrm{P} 3 / 200$ & 67.98 & 99.85 & 1.95 & 198.10 \\
Série 3 & $\mathrm{P} 3 / 300$ & 68.55 & 101.26 & 1.90 & 296.88 \\
(avec connecteurs goujon) & $\mathrm{P} 3 / 400$ & 68.80 & 100.15 & 2.00 & 397.75 \\
& $\mathrm{P} 3 / 500$ & 68.73 & 100.25 & 1.93 & 480.00 \\
\hline Série 4 & $\mathrm{P} 4 / 200$ & 68.62 & 99.22 & 1.90 & 200.00 \\
(avec lien horizontal & $\mathrm{P} 4 / 300$ & 68.58 & 101.70 & 1.95 & 300.75 \\
100 mm) & $\mathrm{P} 4 / 400$ & 69.73 & 102.44 & 2.00 & 401.13 \\
& $\mathrm{P} 4 / 500$ & 68.12 & 102.50 & 1.95 & 490.00 \\
\hline Série 5 & $\mathrm{P} 5 / 200$ & 70.13 & 99.13 & 2.05 & 201.13 \\
(avec lien horizontal & $\mathrm{P} 5 / 300$ & 67.87 & 102.85 & 1.95 & 301.25 \\
50 mm) & $\mathrm{P} 5 / 400$ & 69.43 & 101.00 & 2.05 & 399.50 \\
& $\mathrm{P} 5 / 500$ & 69.88 & 101.83 & 2.05 & 498.17 \\
\hline
\end{tabular}

La capacité portante des profilés composés renforcés par les différents connecteurs est supérieure à la capacité portante des profilés composés sans connecteurs. On remarque que les poteaux composés avec des connecteurs horizontaux ont une capacité plus élevée que les poteaux composés avec connecteurs type goujon et cornière et sans connecteurs, comme l'illustre la "Figure 2". On remarque aussi que plus l'élancement augmente plus la charge de rupture diminue.

Le gain de résistance est plus élevé pour les poteaux composés renforcés avec des liens horizontaux qu'aux poteaux composés sans connecteur et avec connecteurs de cisaillement types cornières et goujons comme le montre la "Figure 3". La capacité portante des profilés sans connecteurs, avec connecteurs cornières et goujon et des liens horizontaux d'espacement $100 \mathrm{~mm}$ et $50 \mathrm{~mm}$ a augmenté respectivement de 51\%, 56\%, 62\%, 125\%, $160 \%$ par rapport aux profilés en acier vide.

Le rapport des charges des poteaux composés avec connecteurs à celles sans connecteurs varie linéairement et augmente avec l'élancement des poteaux composés, comme le montre la "Figure 4". Le gain de résistance des poteaux composés renforcés respectivement par des connecteurs de cisaillement cornières, goujons et des liens horizontaux d'espacement $100 \mathrm{~mm}$ et $50 \mathrm{~mm}$ est de l'ordre de $4 \%, 7 \%, 50 \%$ et $73 \%$ par rapport au poteaux composés sans connecteurs. Ceci illustre bien la 
contribution de l'utilisation des connecteurs et des liens horizontaux dans l'amélioration de la capacité portante des profilés en acier partiellement enrobés.

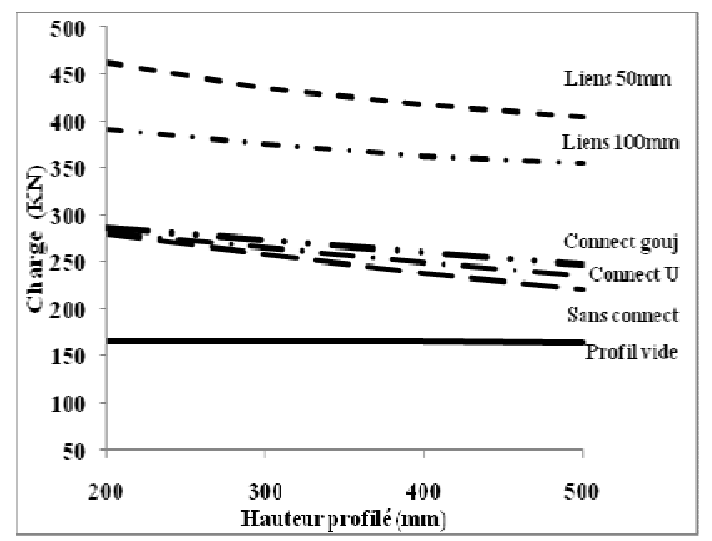

Fig. 2. Charges de rupture expérimentales (Nue) des colonnes composées et vides.

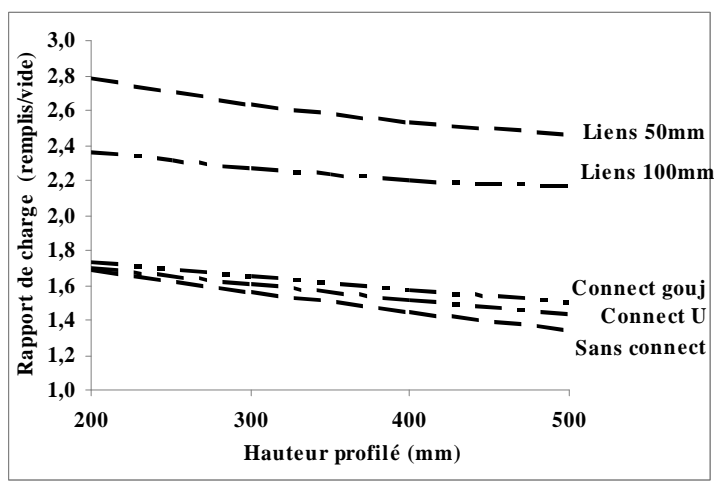

Fig. 3. Gain de résistance en fonction de l'élancement.

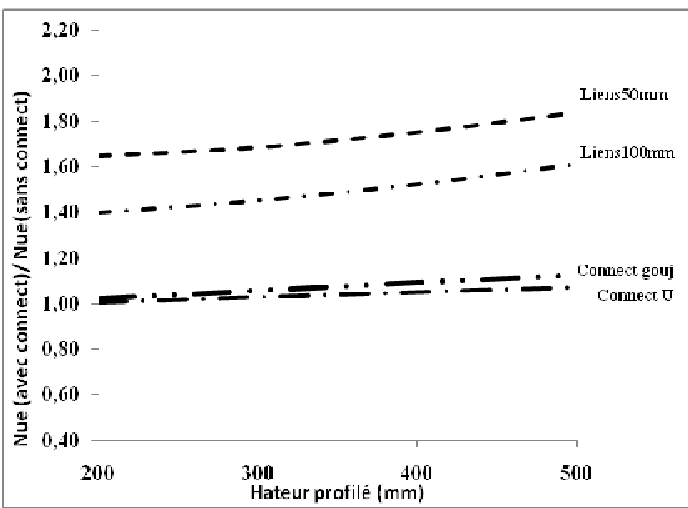

Fig. 4. Rapport de charge des colonnes en acier composées avec connecteurs et sans connecteurs.

La variation du rapport de contrainte pour l'acier et le béton à 28 jours des poteaux composés sans connecteurs et avec connecteurs est montrée sur la "Figure 5". Le rapport de contrainte 
(fc/fc28) varie de 1.00 à 0.49 , de 0.91 à 0.56 , de 0.95 à 0.65 , de 1.43 à 1.21 et de 1.87 à 1.52 respectivement pour les spécimens composés sans connecteurs, les spécimens renforcés avec connecteurs cornières et goujons et les spécimens renforcés par des liens horizontaux d'espacement $100 \mathrm{~mm}$ et $50 \mathrm{~mm}$.

On constate que la contribution du béton à la résistance ultime des profilés en acier composés renforcés par des liens horizontaux est meilleure que celle des profilés en acier composés renforcés par des connecteurs de cisaillement de types cornières et goujon et des profilés sans connecteurs.

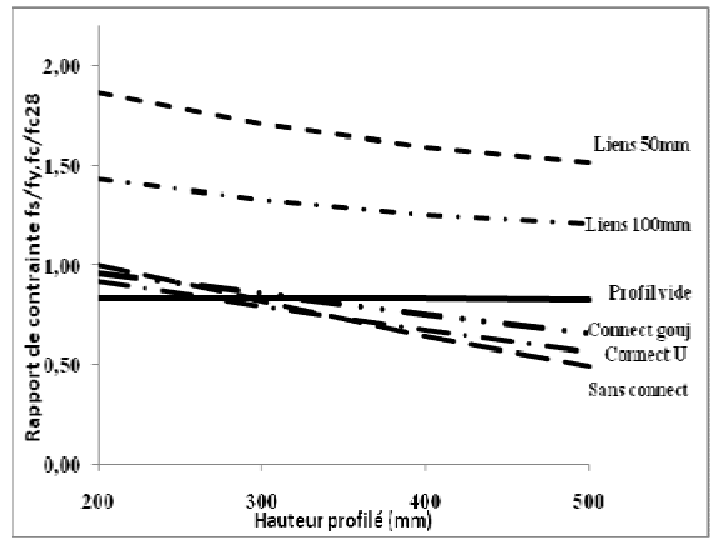

Fig. 5. Rapport de contrainte de l'acier et du béton en fonction de l'élancement.

La "Figure 6" illustre le rapport des charges des poteaux composés à celles calculées selon l'EC4. On remarque que ce dernier donne des valeurs conservatrices pour les profilés renforcés par des liens horizontaux, par contre il donne une prévision excessive pour les profilés composés sans connecteurs et renforcés par des connecteurs de caillement de type cornières et goujons.

L'Eurocode 4 prévoie une capacité inférieure de $9 \%$ à $21 \%$ et $17 \%$ à $29 \%$ par rapport aux résultats d'essais respectivement pour les profilés en acier partiellement remplis renforcés par des liens horizontaux d'espacement $100 \mathrm{~mm}$ et $50 \mathrm{~mm}$. Par ailleurs, il prévoie une capacité supérieure de $8 \%$ à $33 \%, 6 \%$ à $31 \%$ et de $4 \%$ à $22 \%$ comparativement aux résultats d'essais respectivement pour les profilés en acier partiellement remplis sans connecteur et renforcés par des connecteurs cornières et goujons.

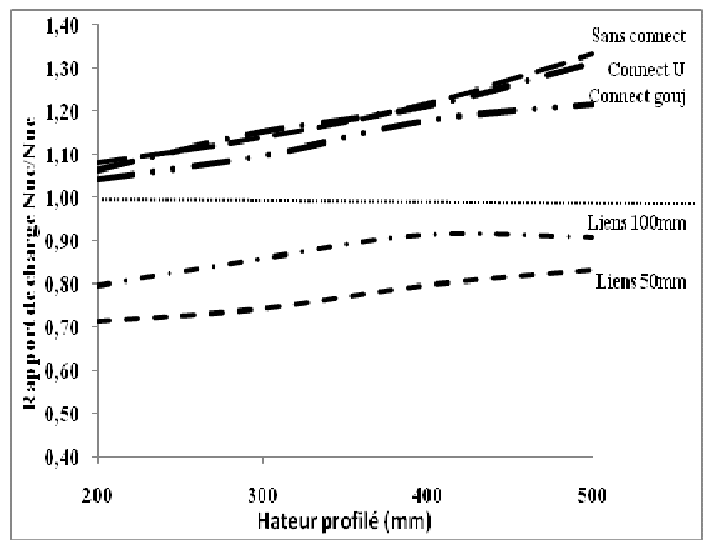

Fig. 5. Rapport de charge (Nuc/Nue) des profilés composés. 


\section{Conclusion}

Les essais effectués sur des poteaux à section en I reconstituée en acier laminé à froid et soudé remplis partiellement de béton ordinaire nous ont permis de faire les conclusions suivantes :

- Une augmentation de la capacité portante de $51 \%, 56 \%, 62,125 \%$ et $160 \%$ a été observée respectivement pour les profilés composés sans connecteurs, renforcés par des connecteurs de caillement de type cornières et goujons et les profilés composés renforcés par des liens horizontaux d'espacement $100 \mathrm{~mm}$ et $50 \mathrm{~mm}$ par rapport aux profilés vides. Dans le même ordre de comparaison, il y a eu également une augmentation de l'ordre de 4\%,7\%,50\% et $73 \%$ par rapport aux poteaux composés sans connecteurs.

- La capacité portante diminue avec l'augmentation de l'élancement pour les profilés vides et composés.

- Les liens en acier horizontaux soudés au niveau des ailes des profilés en acier partiellement enrobés, ont diminué le voilement local de ces derniers. Plus la distance entre les liens est petite plus les semelles résistent au voilement local. Le mode de rupture de ce type de profilés composés est l'écrasement partiel ou total du béton entre les liens horizontaux avec voilement symétrique et dissymétrique des ailes en acier et cassure de la soudure des liens horizontaux.

- L'EC4 prévoie une capacité moyenne supérieure de 19\%, 18\% et 13\% respectivement pour les profilés composés sans connecteurs et avec connecteurs de cisaillements cornières, goujons à celle trouvé expérimentalement. Par contre l'EC 4 est conservateur et donne une capacité moyenne inferieure de $13 \%$ et $23 \%$ pour le cas des profilés en acier composés renforcés par des liens horizontaux d'espacement $100 \mathrm{~mm}$ et $50 \mathrm{~mm}$.

\section{References}

1. R. Tremblay, B. Massicotte, B., I. Filion, R. Maranda, Proc., SSRC Annual Technical Meeting, Atlanta, pp.195-204 (1998)

2. T. Chicoine, R. Tremblay, B.Massicotte, J. Ricles, L.-W. Lu, J. Struct. Eng., 128 (3), pp. 279 287 (2002)

3. R. Bouchereau, J.-D. Toupin, Rep. No. EPM/GCS, Dept. of Civil, Geological, and Mining Engineering, Ecole Poly Technique, Montréal, (2003)

4. M. Begum, M., R. G. Driver, A. E. Elwi, Proc., SSRC/NASCC Joint Annual Stability Conf., Montréal, pp. 241-255 (2005)

5. B.S. PRICKETT, R.G. DRIVER, Structural Engineering Rep 262, University of Alberta, Edmonton (2006)

6. N. Handel, N.-E. Chikh, D. Achoura , International Review of Mechanical Engineering (IREM), 3 n. 6, pp. 825-832 (2009)

7. Y. F Yang, L. H. Han, 2006, Journal of Constructional Steel Research, 62, n. 12, pp. 1310-1324 (2006)

8. C. C. Chen., N. J Lin. , Journal of Constructional Steel Research , 62 , pp. 424-433 (2006)

9. M. Begum, R. G . Driver, M. ASCE, A. E. Elwi, M. ASCE, Using the Dynamic Explicit Method, Journal of Structural Engineering, 133, n. 3, pp. 326-334 (2007)

10. N. Handel, D. Achoura, N.-E. Chikh, $19^{\text {eme }}$ Congrés Français de Mécanique, Marseille, France (2009) 